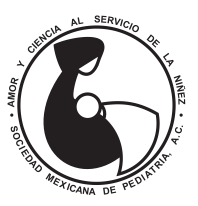

Vol. 87, No. 2 - Marzo-Abril 2020. pp 46-50 https://dx.doi.org/10.35366/94167

Revista Mexicana de

\title{
Efecto de solución salina ionizada nasal pH neutro en la función pulmonar e infecciones respiratorias de niños asmáticos
}

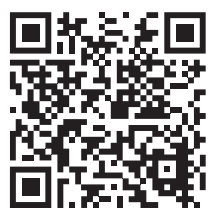

\author{
Eduardo Antonio Lara-Pérez, ${ }^{\ddagger}, *$ Cesar Virgen-Ortega, ${ }^{\S}$ Félix Guillermo Márquez-Celedonio,॥ \\ Josué Elí Villegas-Domínguez, "Celia Nohemí Crespo-Cortés\| \\ * Clínica de Atención de Asma, Academia Mexicana de Pediatría A.C., Veracruz, Veracruz; ${ }^{\S}$ Presidente del Colegio de Pediatría \\ de Tabasco; "Facultad de Medicina "Dr. Porfirio Sosa Zárate”, Universidad del Valle de México, Veracruz, México.
}

\begin{abstract}
RESUMEN
Objetivo: Determinar el efecto de administración de solución salina ionizada nasal sobre el flujo inspiratorio máximo nasal (FIMN), flujo espiratorio máximo (FEM) y frecuencia de infecciones de tracto respiratorio superior (ITRS) en niños y adolescentes asmáticos. Material y métodos: Estudio cuasi-experimental, evaluando la evolución antes y después, de pacientes con asma mal controlada, y sin diagnóstico de rinitis. Durante cuatro meses de tratamiento con solución salina ionizada nasal se evaluó el FIMN, FEM y la frecuencia de ITRS. Análisis estadístico: $\chi^{2}$ con corrección de Yates, $U$ de Mann-Whitney, Wilcoxon y Friedman. Resultados: Se incluyeron 80 asmáticos de $8.7 \pm 2.1$ años. FIMN fue de $58.4 \pm 19.3 \mathrm{Lpm}$ en la medición basal y de $104.7 \pm 29.2$ a los cuatro meses $(p<0.05)$, FEM basal fue de $192.7 \pm 58.7$ $y$ de $222.3 \pm 67.0 \mathrm{Lpm}$, al final del seguimiento $(p<0.05)$. La frecuencia de ITRS disminuyó progresivamente, pasando de $88.8 \%$ en el primer mes a ningún caso en el cuarto mes $(p<0.05)$. Conclusiones: La solución salina nasal ionizada en asmáticos sin diagnóstico de rinitis parece mejorar en FIMN, FEM y la frecuencia ITRS, sin embargo, se requieren de ensayos clínicos aleatorizados para demostrar su verdadera efectividad.
\end{abstract}

Palabras clave: Asma, flujo inspiratorio máximo nasal, flujo espiratorio máximo, infecciones respiratorias de vías aéreas superiores, solución salina ionizada.

\section{ABSTRACT}

Objective: To determine the effect of administration of nasal ionized saline solution on nasal maximum inspiratory flow (NMIF), maximum expiratory flow (MEF) and frequency of upper respiratory tract infections (URTI) in asthmatic children. Material and methods: Quasi-experimental study, evaluating the evolution, before and after, of patients with poorly controlled asthma and without rhinitis. During four months of treatment with nasal ionized saline, the NMIF, MEF and the frequency of URTI were evaluated. Statistical analysis: $\chi 2$ with Yates correction, Mann-Whitney $U$, Wilcoxon and Friedman. Results: Eighty asthmatic children aged $8.7 \pm 2.1$ years were included. NMIF of $58.4 \pm 19.3$ Lpm at baseline and $104.7 \pm 29.2$ at four months $(p<0.05)$, baseline MEF was $192.7 \pm 58.7$ and $222.3 \pm 67.0 \mathrm{Lpm}$ at the end of follow-up $(p<0.05)$. URTI frequency decreased progressively, $88.8 \%$ in the first month to any case in the fourth month $(p<0.05)$. Conclusions: Ionized nasal saline in asthmatics without a rhinitis appears to improve FIMN, FEM, and ITRS frequency, however, randomized clinical trials are required to demonstrate its effectiveness.

Keywords: Asthma, peak nasal inspiratory flow, peak expiratory flow, upper respiratory tract infections, ionized saline solution.

\footnotetext{
* Correspondencia: EALP, lape5104@hotmail.com

Conflicto de intereses: Los autores declaran que no tienen.

Citar como: Lara-Pérez EA, Virgen-Ortega C, Márquez-Celedonio FG, Villegas-Domínguez JE, Crespo-Cortés CN. Efecto de solución salina ionizada nasal pH neutro en la función pulmonar e infecciones respiratorias de niños asmáticos. Rev Mex Pediatr. 2020; 87(2):46-50. doi: $10.35366 / 94167$

[Effect of $\mathrm{pH}$ nasal ionized saline solution on pulmonary function and respiratory infections of asthmatic children]
} 


\section{INTRODUCCIÓN}

La rinitis y el asma son padecimientos frecuentes a nivel mundial, su prevalencia alcanza tasas de 10 a $37.6 \%{ }^{1-4} \mathrm{El}$ asma se manifiesta clínicamente por limitación variable del flujo espiratorio que acompaña los síntomas típicos de sibilancias, disnea, opresión de pecho y tos. Las infecciones respiratorias, especialmente virales, además de otros factores pueden desencadenar la presentación o exacerbación de los síntomas. ${ }^{5}$ Además de la inflamación, los componentes fisiopatológicos del asma son espasmo bronquial e hipersecreción de moco. El exceso de moco no sólo obstruye las vías respiratorias, sino que contribuye a la hipersensibilidad, y es indicativo de un control pobre de la enfermedad. ${ }^{6}$ Además de las infecciones de repetición de vías aéreas, se ha demostrado relación de comorbilidad entre rinitis y asma. ${ }^{7-12}$

Como parte de la evaluación de los niños con asma, la vigilancia de la función pulmonar es necesaria para adecuar el manejo mediante la medición del volumen espiratorio forzado en un segundo (FEV1) y del flujo espiratorio máximo (FEM) que permiten establecer el estado del control del paciente, ${ }^{13}$ lo cual puede complementarse evaluando el flujo inspiratorio máximo nasal (FIMN). ${ }^{14,15}$

Los objetivos del manejo del asmático se orientan a alcanzar un control adecuado de los síntomas, disminuir el riesgo de exacerbaciones, preservar la función pulmonar, reducir la limitación del flujo de aire y de efectos secundarios de los medicamentos. ${ }^{1} \mathrm{El}$ tratamiento incluye medicamentos de mantenimiento y de rescate para las exacerbaciones. ${ }^{1,16}$ Por su parte, la irrigación nasal con solución salina ha mostrado eficacia en niños con congestión nasal por infecciones, enfermedades alérgicas o rinosinusitis. ${ }^{17-19} \mathrm{El}$ manejo de la respiración nasal en asma, especialmente si se acompaña de rinitis, podría representar beneficios para el curso clínico y calidad de vida del paciente.

El presente estudio tiene el propósito de evaluar el efecto de una solución electrolizada de superoxidación con $\mathrm{pH}$ neutro en la función pulmonar medida a través de FEM y FIMN y frecuencia de infecciones respiratorias del tracto respiratorio superior (ITRS) de asmáticos con mal control.

\section{MATERIAL Y MÉTODOS}

Se trata de un ensayo clínico, cuasi-experimental, evaluando la evolución antes y después de aplicar la intervención, que se realizó de octubre de 2017 a marzo de 2018. Se incluyeron niños y adolescentes, de ambos sexos, con al menos un año de diagnóstico establecido de asma bronquial, la cual se encontraba mal controlada a pesar del tratamiento. Estos pacientes no tenían diagnóstico previo de rinitis y presentaban cuadros de ITRS intercurrentes.

El diagnóstico de asma se estableció de acuerdo con los criterios de la actualización 2017 de la Global Strategy for Asthma Management and Prevention de la Global Initiative for Asthma (GINA) ${ }^{1}$, en particular en quienes se confirmó limitación variable del flujo espiratorio con FEM. En todos los casos se descartaron otros padecimientos, como fibrosis quística, bronquiectasias, cardiopatía congénita, presencia de cuerpos extraños en vías aéreas superiores y disquinesia ciliar primaria.

El estatus de mal control se identificó por la presencia, en las cuatro semanas previas, de tres de los cuatro síntomas siguientes: síntomas diurnos de asma más de dos veces por semana, vigilias durante las noches debido a la enfermedad, necesidad de aplicación de medicación para los síntomas más de dos veces por semana y limitación de la actividad física.

La evaluación inicial incluyó el registro de edad y sexo, además de realizar mediciones antropométricas con báscula Detecto ${ }^{\circledR}$ y estadímetro. El FIMN se midió con un flujómetro en escala de litros por minuto (Lpm) con mascarilla nasofacial (MD Instruments ${ }^{\circledR}$ ), y el FEM con un flujómetro en escala de litros por minuto marca TruZone ${ }^{\circledR}$, requiriendo al menos 30 minutos de reposo después de haber realizado esfuerzo físico, o después de haber tomado bebidas calientes o estimulantes. Las mediciones de FEM y FIMN se realizaron antes de iniciar la intervención terapéutica y cada mes durante cuatro meses estando el paciente de pie, con cinco intentos en cada ocasión para cada flujo, tomando la calificación más alta como registro. Los valores obtenidos se compararon con respecto de los niños sanos que viven a nivel del mar, de acuerdo con sexo y estatura. ${ }^{20}$

Después de la evaluación inicial, a todos los pacientes se indicó la administración por vía nasal de una solución electrolizada de superoxidación con $\mathrm{pH}$ neutro y especies activas de cloro y oxígeno al $0.0015 \%$. Se indicó la administración en posición vertical de dos disparos de la solución en cada narina dos veces al día, pero con la instrucción de realizarla a libre demanda hasta cuatro aplicaciones por día, de acuerdo al estado de hipersecreción nasal. Las aplicaciones se realizaron todos los días de la semana por cuatro meses. Los pacientes fueron vigilados mensualmente durante cuatro meses por el mismo investigador, en horario de 11 a 19 horas, para determinar el estado de control del asma, presencia de infecciones, así como para medir FIMN y FEM. 


\begin{tabular}{|c|c|}
\hline & $\mathrm{n}(\%)$ \\
\hline \multicolumn{2}{|l|}{ Edad (años) } \\
\hline $6-7$ & $26(32.5)$ \\
\hline $8-9$ & $29(36.3)$ \\
\hline $10-11$ & $18(22.5)$ \\
\hline $12-13$ & $5(6.3)$ \\
\hline $14-15$ & $2(2.5)$ \\
\hline \multicolumn{2}{|l|}{ Sexo } \\
\hline Femenino & $32(40.0)$ \\
\hline Masculino & $48(60.0)$ \\
\hline Talla & $130.9 \pm 12.6$ \\
\hline
\end{tabular}

Análisis estadístico. Los datos cualitativos se presentan como frecuencias absolutas y relativas. Los cuantitativos con promedio \pm desviación estándar (DE). El análisis inferencial se efectuó con $\chi^{2}$ con corrección de Yates, U de Mann-Whitney, y pruebas de Wilcoxon y Friedman. El nivel de significancia estadística fue $<0.05$. Los análisis se realizaron con el software Statistical Package for the Social Sciences (SPSS) versión 25.0.

Aspectos éticos. La inclusión de los participantes fue con firma de consentimiento informado por los padres o tutores. Antes del inicio del estudio el protocolo fue autorizado por el Comité de Investigación y Bioética de la Universidad del Valle de México, campus Veracruz.

\section{RESULTADOS}

Se incluyeron 80 pacientes con promedio de edad de 8.7 \pm 2.1 años, talla de $130.9 \pm 12.6 \mathrm{~cm}$, siendo 48 (60.0\%) del sexo masculino (Tabla 1). Los pacientes que presentaron ITRS en el mes de la medición basal fueron 64 (80.0\%). En la medición basal, el FEM se encontró en $181.8 \pm 55.6 \mathrm{Lpm}$, el cual, en general (66.3\%), correspondió al valor de referencia normal. En los hombres el FEM fue de $184.1 \pm 53.3 \mathrm{Lpm}$ y en las mujeres de $178.3 \pm 59.4 \mathrm{Lpm}$.

Por su parte el FIMN inicial en el total de la muestra fue de $58.4 \pm 19.3 \mathrm{Lpm}$, subiendo a $75.9 \pm 22.0 \mathrm{Lpm}$ después de un mes de iniciado el tratamiento, y 104.7 $\pm 29.2 \mathrm{Lpm}(\mathrm{p}<0.001)$ al finalizar los cuatro meses de intervención, con un incremento total de 79.3\% (Tabla 2). Cuando se analizaron los datos por sexo (Figura 1), los pacientes masculinos mostraron cierta tendencia a tener mejor evolución que las mujeres; los masculinos presentaron $58.6 \pm 19.5 \mathrm{Lpm}$ de FIMN en la evaluación basal y $110.9 \pm 29.6 \mathrm{Lpm}$ al final de la intervención, con un incremento de 89.3\% ( $\mathrm{p}<0.001)$. Mientras que en las pacientes de sexo femenino la medición de inicio fue de $58.0 \pm 19.2$, y $95.3 \pm 26.3 \mathrm{Lpm}$ después de cuatro meses de tratamiento, con incremento de $64.3 \%(\mathrm{p}<0.001)$.

Como también se muestra en la Tabla 2, el FEM evolucionó de $181.8 \pm 55.6$ Lpm en la medición inicial a $192.7 \pm 58.7$ con un mes de intervención experimental y $222.3 \pm 67.0$ al término de cuatro meses de tratamiento $(\mathrm{p}<0.001)$. En la medición basal de FEM, 45 pacientes (56.3\%) se encontraban entre 50 y $69 \%$ del valor de referencia normal, mientras que después de cuatro meses de intervención 63 (78.8\%) estaban en intervalo de 70 a $89 \%$ y $12(15.0 \%)$ con valor superior a $90 \%$.

Tabla 2: Evolución del flujo inspiratorio máximo nasal, espiratorio máximo e infecciones

respiratorias en niños manejados con solución electrolizada de pH neutro, $\mathrm{N}=80$.

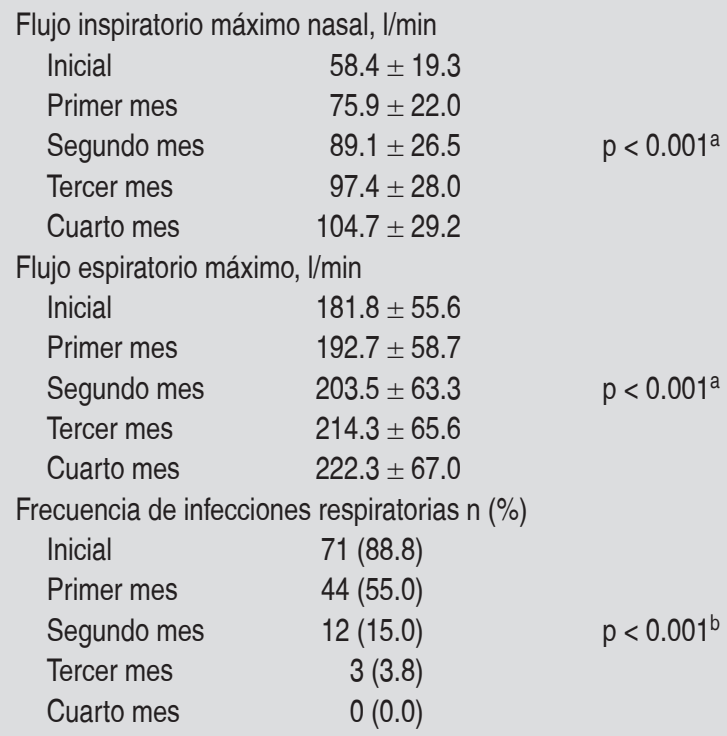

Valores expresados en media \pm desviación estándar 0 valor absoluto y frecuencia relativa.

a Valores de $p$ obtenidos con prueba de Friedman.

${ }^{b}$ Valores de $p$ obtenidos con prueba $\chi^{2}$ con corrección de Yates. 


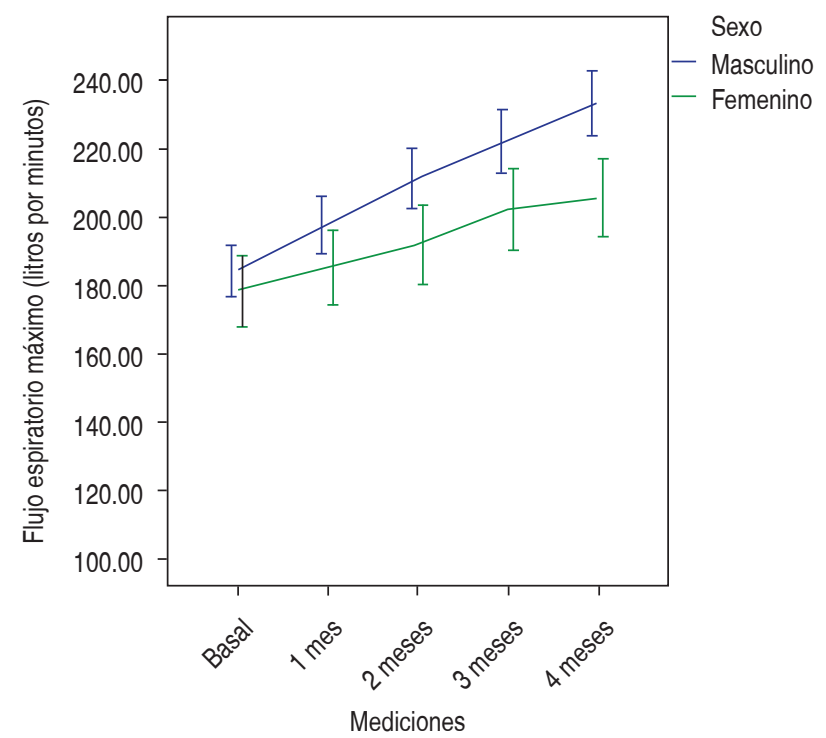

Figura 1: Evolución del flujo espiratorio máximo en niños y niñas que recibieron por vía nasal solución electrolizada de $\mathrm{pH}$ neutro por cuatro meses.

En el FEM, los varones tuvieron un comportamiento mejor que las mujeres (Figura 2). En los primeros, la medición basal fue de $184.1 \pm 53.3 \mathrm{Lpm}, \mathrm{y}$ de $233.3 \pm$ $65.6 \mathrm{Lpm}$ al término de la intervención $(\mathrm{p}<0.001)$. En las mujeres, pasó de $178.3 \pm 59.4 \mathrm{Lpm}$ al inicio a 205.6 \pm 66.5 al final de los cuatro meses de tratamiento ( $p$ $<0.001)$.

Con respecto a las ITRS, la frecuencia de casos a lo largo de los cuatro meses se muestra en la Tabla 2. Como se observa, progresivamente hubo disminución hasta no observar casos en el último mes de vigilancia.

\section{DISCUSIÓN}

Los hallazgos de nuestro estudio mostraron que una solución electrolizada nasal de superoxidación con $\mathrm{pH}$ neutro en escolares y adolescentes con asma bronquial mejoró significativamente el FIMN y el FEM. Además, se observó disminución de la frecuencia de ITRS. El FIMN presentó un incremento de $79 \%$ después de cuatro meses de tratamiento comparado con el valor inicial, mientras que el incremento del FEM fue de $22.3 \%$. La notable mejoría del FIMN tiene trascendencia clínica al representar un decremento significativo de la obstrucción aérea en el segmento nasal, lo cual puede contribuir dada su correlación con el FEM en la mejoría de la función pulmonar del paciente asmático.

Los resultados también mostraron que durante la intervención con solución electrolizada de superoxidación con $\mathrm{pH}$ neutro, los casos de ITRS se redujeron, lo cual puede estar relacionado con la disminución de la hipersecreción de moco, que constituye una característica en la rinitis alérgica y el asma bronquial, y que contribuye a su morbilidad, ${ }^{6} \mathrm{o}$ bien, a sus propiedades bactericidas y reducción de la multiplicación viral, demostrada en cultivos celulares. ${ }^{18}$ Se debe tener en cuenta que el asma, rinitis e ITRS presentan variación estacional, lo cual tiene que considerarse al interpretar nuestros resultado. Este estudio se realizó durante otoño e invierno del hemisferio.

Si bien, los resultados de nuestra investigación tienen la fortaleza de proceder de una intervención terapéutica evaluada prospectivamente; no obstante, tiene las limitaciones inherentes a los estudios experimentales, sin cegamiento y sin grupo control. Hermelingmeier y colegas ${ }^{17}$ identificaron en una revisión sistemática y metaanálisis que las irrigaciones nasales de solución salina en la rinitis alérgica redujeron $27.7 \%$ los síntomas, $61.1 \%$ el consumo de medicamentos y mejoraron la calidad de vida en $27.9 \%$; sin embargo, en dicha revisión no incluyeron estudios en pacientes asmáticos. Ante esto último, es necesario recordar que otra limitante de nuestro estudio es que no se realizó el diagnóstico de rinitis alérgica.

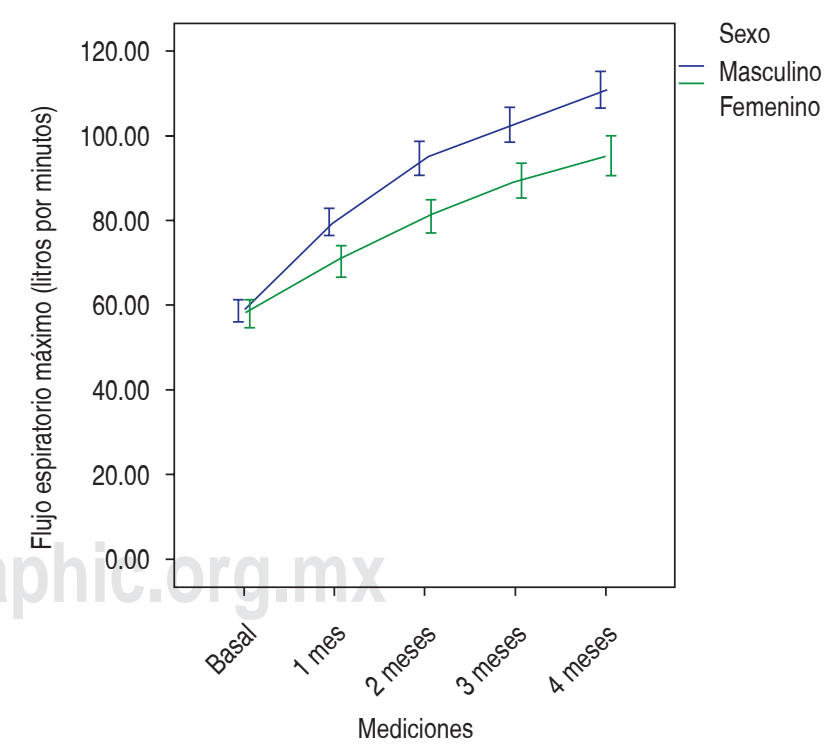

Figura 2: Evolución del flujo inspiratorio máximo nasal en niños y niñas que recibieron por vía nasal solución electrolizada de $\mathrm{pH}$ neutro por cuatro meses. 
Desde el punto de vista clínico, nuestros hallazgos consideran que en pacientes con asma, la mejoría de la función nasal mediante irrigaciones nasales de solución electrolizada de superoxidación puede servir como medida adyuvante del tratamiento. Este hallazgo es concordante con estudios previos. ${ }^{15}$

\section{CONCLUSIONES}

La administración por irrigación nasal de solución electrolizada de superoxidación con $\mathrm{pH}$ neutro parece tener efectos benéficos en niños asmáticos, ya que coadyuva en la recuperación del FIMN y el FEM, y posiblemente sobre las ITRS; sin embargo, se requieren de ensayos clínicos aleatorizados para determinar su verdadera efectividad.

\section{REFERENCIAS}

1. Pearce N, Aït-Khaled N, Beasley R, Mallol J, Keil U, Mitchell E, et al Worldwide trends in the prevalence of asthma symptoms: phase III of the International Study of Asthma and Allergies in Childhood (ISAAC). Thorax. 2007; 62: 758-766.

2. Lai CKW, Beasley R, Crane J, Foliaki S, Shah J, Weiland S, the ISAAC Phase Three Study Group. Global variation in the prevalence and severity of asthma symptoms: phase three of the International Study of Asthma and Allergies in Childhood (ISAAC). Thorax. 2009; 64: 476-483.

3. Kim JL, Brisman J, Al Äberg M, Forslund HB, Winkvist A, Torén $\mathrm{K}$. Trends in the prevalence of asthma, rhinitis, and eczema in 15-year-old adolescents over an 8-year period. Respiratory Medicine. 2014; 108: 701-708.

4. Lara-Pérez EA. Prevalencia del asma infantil al nivel del mar. Rev Alerg Asma e Inmunol Pediatr. 2001; 10(3): 88-92.

5. Global Initiative for Asthma. Global Strategy for Asthma Management and Prevention, 2017.

6. Evans CM, Kim K, Tuvim MJ, Dickey BF. Mucus hypersecretion in asthma: causes and effects. Curr Opin Pulm M. 2009; 15(1): 4-11.

7. Chiron R, Vachier I, Khanbabaee G, Molinari N, Varrin M, Godard $\mathrm{P}$, Chanez P. Impact of rhinitis on asthma control in children: association with FeNO. J Asthma. 2010; 47: 604-608.
8. Kariyawasam HH, Rotiroti G. Allergic rhinitis, chronic rhinosinusitis and asthma: unravelling a complex relationship. Curr Opin Otolaryngol Head Neck Surg. 2013; 21: 79-86.

9. De Oliveira TB, Klering EA, da Veiga ABG. Is recurrent respiratory infection associated with allergic respiratory disease? J Asthma. 2018; 13: 1-7.

10. Pite $\mathrm{H}$, Pereira AM, Morais-Almeida $\mathrm{M}$, Nunes $\mathrm{C}$, Bousquet $\mathrm{J}$, Fonseca JA. Prevalence of asthma and its association with rhinitis in the elderly. Respir Med. 2014; 108: 1117-1126.

11. Luna M de F, Fisher GB, Luna JR, Silva MG, Almeida PC, Chiesa D. Prevalence of rhinitis among 6 and 7-year old students in Fortaleza. Rev Assoc Med Bras. 2014; 60: 357-64.

12. García-García ML, Calvo C, Ruiz S, Pozo F, Del Pozo V, Remedios $\mathrm{L}$ et al. Role of viral coinfections in asthma development. PloS One. 2017; 12(12): e0189083.

13. Moeller A, Carlsen KH, Sly PD, Baraldi E, Piacentini G, Pavord I et al. Monitoring asthma in childhood: lung function, bronchial responsiveness and inflammation. Eur Respir Rev. 2015;24:204-215.

14. Mohan S, Fuller JC, Ford SF, Lindsay LW. Diagnostic and therapeutic management of nasal airway obstruction: advances in diagnosis and treatment. JAMA Facial Plast Surg. 2018; 20(5): 409-418.

15. Pite H, Pimenta L, Henriques AC, Marques I, Camarinha C, Laurenco $\mathrm{AV}$ et al. Lower airway flow influences peak nasal inspiratory flow in school-aged children. Rhinology. 2018; 56(3): 288-296.

16. Choby GW. Pharmacotherapy for the treatment of asthma: current treatment options and future directions. Int Forum Allergy Rhinol. 2015; 5(S1): S35-S40.

17. Hermelingmeier KE, Weber RK, Hellmich M, Heubach CP, Mösges R. Nasal irrigation as an adjunctive treatment in allergic rhinitis: a systematic review and meta-analysis. Am J Rhinol Allergy. 2012; 26(5): 119-125.

18. Cabello-Gutiérrez C, Rosete-Olvera P, Manjarrez-Zavala ME. Efecto de una solución electrolizada de superoxidación con pH neutro sobre la infección del virus de influenza A en células MDCK. Rev Inst Nal Enf Resp Mex. 2009; 22: 280-287.

19. Medina-Tamayo J, Sánchez-Miranda E, Belleza-Tapia H, Ambriz X, Cid ME, González-Espinosa D, Gutiérrez SS, GonzálezEspinosa C. Super-oxidized solution inhibits IgE-antigeninduced degranulation and cytokine release in mast cells. Int Immunopharmacol. 2007; 7(8): 1013-1024.

20. Lara-Pérez EA. Peak expiratory flow at sea level. Int Pediatr. 2001; 16: 89-93. 